\title{
ATTENTIONAL BIASES IN PATIENTS WITH ALCOHOL DEPENDENCE: INFLUENCE OF CO- EXISTING PSYCHOPATHOLOGY
}

Julia MA Sinclair * Matthew Garner, ${ }^{1,4}$, Sonja C Pasche ${ }^{2}$, Thomas B Wood ${ }^{3}$, David S Baldwin ${ }^{1,2}$

1. Clinical and Experimental Sciences, Faculty of Medicine, University of Southampton

2. Department of Psychiatry and Mental Health, University of Cape Town, South Africa

3. GP ST3, Wessex /general Practice Vocational Training Scheme

4. Psychology, Faculty of Social and Human Sciences, University of Southampton

\author{
* Address for correspondence \\ Julia MA Sinclair \\ Associate Professor in Psychiatry \\ University Department of Psychiatry \\ Academic Centre, College Keep \\ 4-12 Terminus Terrace, \\ Southampton SO14 3DT \\ Tel. +442380718520 \\ Fax +442380718533 \\ julia.sinclair@soton.ac.uk
}

Word count: 3101

\section{Declaration of interest}

The study was funded by a grant from the Research Management Committee of the University of Southampton. SCP was supported through the European Union Marie Curie (People) International Research Staff Exchange Scheme (PIRSES-GA-2010-269213: 'EUSARNAD'). No author has any link with any tobacco, alcohol or gaming industry. JMAS has been part of an international advisory board (nalmefene) for $\mathrm{H}$. Lundbeck A/S. 


\section{ABSTRACT}

Objective: The effects of coexisting psychopathology on disorder-specific attentional biases in patients with alcohol dependence are uncertain. We undertook a cross-sectional study assessing attentional biases to alcohol-, depression- and anxiety- related stimuli using the visual probe task (VPT) in patients with alcohol dependence, attending a community alcohol service.

Methods: Using the VPT, we presented disorder-specific words (relating to alcohol, anxiety and depression) for $500 \mathrm{~ms}$ and measured reaction times.

Results: Participants demonstrated a significant attentional bias towards alcohol-related cues (mean 8.5, $\mathrm{p}=0.03$ ) but significant avoidance of depression-related cues (mean -8.4, p=0.01). The sub-group of participants who were recently abstinent $(n=70)$ showed greatest avoidance of depression-related cues $(\mathrm{t}(69)=2.68, \mathrm{p}<0.01)$, but no significant vigilance towards alcohol or anxiety cues: whereas those still drinking $(\mathrm{N}=43)$ showed attentional biases towards alcohol-related $(\mathrm{t}(42)=2.70, \mathrm{p}=0.01)$ and social anxiety related cues $(\mathrm{t}(42)=2.84, \mathrm{p}<0.01)$. In the whole sample the magnitude of attentional bias to alcohol was not correlated with length of drinking history, number of comorbid conditions, nor severity of anxiety/depression. Conclusions: In a clinical sample of alcohol dependent patients, further investigation is required to explore whether these attentional biases reflect current drinking status or factors indicating prognosis.

(195 words)

Keywords: alcohol dependence, anxiety, cognitive bias, co-morbidity, depression, visual probe task. 


\section{Background}

Attentional biases (i.e. the tendency to selectively attend to disorder-specific, as compared to neutral, stimuli) may represent key cognitive factors in the aetiology and maintenance of a number of mental disorders, including mood and anxiety disorders (Donaldson et al., 2007; Grant and Beck, 2006), as well as in pain (Moore, 2009). Experimental studies have established the reliability of disorder-specific attentional biases in alcohol use disorders (AUDs) including alcohol dependence (Weinstein and Cox, 2006) with a potentially important role of selective attention in the origin and maintenance of problem drinking (Field et al., 2007; Field and Eastwood, 2005) and in the experience of craving following alcohol abstinence (Field et al., 2013) but the suggestion that altering attentional biases may be beneficial in treatment remains only tentative (Christiansen et al., 2015).

The inconsistent results in this area may be due in part because much research in this field has been conducted in non-clinical populations (Cox et al., 2007; Fadardi and Cox, 2009) and investigations which have been performed in samples of patients with AUDs have tended to either exclude patients with co-morbid conditions or to ignore the potential impact of comorbidity (Sinclair et al., 2010). A systematic review of 17 studies of attentional biases in patients with AUDs found that 10 reports either did not mention or made only minimal statements regarding possible co-morbid conditions (including other substance use disorders); five studies excluded patients with 'psychiatric diagnoses' (variously defined, e.g. 'patients with symptoms of mental illness were excluded'); and two studies excluded patients currently prescribed 'psychotropic medication' (Sinclair et al., 2010).

As such, despite the high prevalence of co-morbidity in AUDs (Grant et al., 2004; Kessler et al., 2005; Cranford et al., 2011; Lingford-Hughes et al., 2012) and probable complex interactions between disorders (Burns et al., 2005; Crum et al., 2012; Bolton et al., 2009; Bruce et al., 2005), little is known about how attentional biases to disorder-specific cues may co-vary with each other, or the impact this may have on the natural history of the condition, or how comorbidity may influence the mechanisms of action of current and novel psychological and pharmacological treatments. Studies in patients with anxiety disorders have demonstrated that co-morbid depression can increase (Kaplan et al., 2006) or reduce (Musa et al., 2003) dysfunctional biases in cognitive/affective processing. It seems reasonable to ask whether a similar process operates in patients with AUDs: and if so, how coexisting psychopathology moderates disorder-specific biases in AUD. To our knowledge no reported study has used disorder-specific tests to measure attentional bias with reference to alcohol use, mood and anxiety disorders in a sample of patients with current or previous alcohol dependence.

We examined attentional biases in a cross-sectional sample of treatment-seeking patients with alcohol dependence, and explored the impact of key variables (length of AUD, presence of comorbid conditions, and current drinking status) on alcohol-specific attentional biases. We also measured the degree of disorder-specific attentional biases (for depression, panic and social anxiety relevant material) in a clinical sample known to have high prevalence of comorbidity with these disorders, and examined the influence of drinking status on these. We hypothesised there would be significant disorder-specific attentional biases (for alcohol, 
depression and anxiety), and that these would be less marked in participants who had achieved abstinence. As an earlier study (Loeber et al., 2009) of alcohol attentional biases (using a visual probe task, VPT) found a significant negative correlation between duration of alcohol dependence and positive attentional biases to alcohol, we included this as a variable to be examined.

\section{Methods}

The study received approval from an NHS research ethics committee (REC ref:08/H0502/40). Patients were recruited from a specialist community alcohol service. Individuals were initially approached by the clinical team, and those who expressed an interest in potential study participation were contacted by the research team. All patients were potentially eligible for participation in the study, regardless of the current phase of treatment.

Recording of demographic and clinical information (age, gender, marital/relationship status, employment status, highest educational qualification reached, number of years of alcohol misuse/dependence, family history of alcohol dependence, current use of alcohol [units/week], and number of previous detoxifications) was followed by assessment with the Mini International Neuropsychiatric Interview (MINI) (Lecrubier et al., 1997; Sheehan et al., 1998), a brief structured interview used to identify the presence of DSM-IV- and ICD-10defined mental disorders: it has acceptable validity and reliability in comparison to similar interview schedules such as the Composite International Diagnostic Interview (CIDI) (Robins et al., 1988). Social anxiety and avoidance was assessed with the Liebowitz Social Anxiety Scale (LSAS) (Liebowitz, 1987), a 24-item questionnaire for assessing the range of social interaction and performance situations that individuals with social anxiety/social phobia may fear and/or avoid: total LSAS scores of 30 or higher indicate social anxiety symptoms of possible or probable clinical significance. Anxiety and depressive symptoms were assessed with the Hospital Anxiety and Depression Scale (HADS) (Zigmond, 1983), a self-report 14-item questionnaire, comprising two sub-scales assessing anxiety (7 items) and depression (7 items): cut-off values of 7 or less on either sub-scale indicate probable non-cases; 8-10, possible cases; and 11 or higher, probable cases of anxiety or depression. The HADS has proven validity, reliability and sensitivity to change in community and clinical samples.

An established computerised visual probe test (MacLeod et al., 1986) was used to measure selective attention to the following word categories: alcohol, social anxiety, depression, panic, and positive ( 8 words per category). Each disorder-specific word was paired with a neutral word of similar length, structure and usage. Participants sat at a table with a laptop (viewing distance $=50 \mathrm{~cm}$ away) in a darkened room to minimise distraction. They were presented with pairs of words (disorder-specific, neutral) on the screen, one pair at a time. Each trial began with presentation of a central fixation cross for $500 \mathrm{~ms}$ after which two words were presented to the left or right of fixation for $500 \mathrm{~ms}$ (font $21 \mathrm{pt}$, Times Roman, average visual angle at $50 \mathrm{~cm}=4.5$ degrees). Immediately following word pair-offset the target probe ( $\uparrow$ or $\downarrow$ arrow) was presented in the location of one of the words. The participants' task was to classify the direction of the target probe by pressing the corresponding button on a Cedrus response box, as quickly and accurately as possible. Participants undertook ten practice trials (comprising 
unique neutral-neutral word pairs) followed by 160 randomized experimental trials (32 trials per word category). Given the aim of the study was to examine a number of potential comorbidities and their interaction in a clinical sample, a balance between words per category and response burden was made. Word location, target probe location and target probe type were counterbalanced within each word category. The task was presented using Inquisit software (v2, Millisecond.com). Previous studies using the VPT have found that the direction and magnitude of alcohol attentional biases differ, depending on whether the stimuli were presented for $50 \mathrm{~ms}$ (Noel et al., 2006; Loeber et al., 2009) or $500 \mathrm{~ms}$ (Townshend and Duka, 2007; Stormark et al., 1997; Vollstadt-Klein et al., 2009) We therefore chose to index attention at $500 \mathrm{~ms}$ because it is the most widely used index of selective attention in studies of alcohol, mood and anxiety (Koster et al., 2004), and bias scores at 500ms strongly correlate with other measures of initial orienting (i.e. speed and direction of the first eye-movement in this task).

Consistent with previous visual probe studies, data from trials in which the participant made an incorrect response were removed, as were anticipatory responses (Reaction Times [RTs] < $100 \mathrm{~ms}$ ) and slowed responses (RTs $>2000 \mathrm{~ms}$ ). For each participant RTs greater than 2.5 standard deviations above their individual mean RT were also removed. Remaining RTs were used to calculate attentional bias scores for each disorder-specific word category. 'Congruent' trials were defined as trials where the visual probe was in the same location as the disorderspecific cue word. In 'Incongruent' trials the visual probe appeared behind the neutral word. Attentional bias scores for disorder-specific words (alcohol, depression, panic, positive, social anxiety) were calculated for each participant by subtracting the mean response time (ms) in congruent trials from the mean response time $(\mathrm{ms})$ in incongruent trials. Positive bias scores reflect greater attention towards disorder-specific words.

\section{Results}

The final sample comprised 113 treatment-seeking patients (Table 1). Sixty-three percent of the sample was male, approximately $20 \%$ of participants were in employment, and the majority (67\%) had a family history of alcohol use disorders. $62 \%$ of patients $(\mathrm{N}=70)$ were in the relapse prevention phase of treatment and currently abstinent from alcohol; but the remaining participants were still drinking, with a median consumption of 70 units per week. Two participants no longer met diagnostic criteria for an alcohol use disorder as they had been abstinent for over a year, but still defined themselves as 'in recovery' from alcohol dependence (see Table 1). Over $80 \%$ of participants met criteria for one or more additional (current) psychiatric disorders as assessed by the MINI: $68 \%$ with diagnoses on the depressive disorders spectrum; $67 \%$ with one or more anxiety disorder, and $17 \%$ with an additional substance use disorder. The mean number of comorbid diagnoses (in addition to alcohol dependence) was three.

\section{Insert Table 1 about here}

Abstinent patients had significantly lower scores on the HADS depression sub-scale, when compared to patients who were still drinking (mean 5.9 vs. 8.3; $\mathrm{t}(111)=2.5, \mathrm{p}=0.01, \mathrm{~d}=$ 0.49 ); and had a longer history of alcohol use disorders ( 17.1 vs. 12.7 years; $t\left(\begin{array}{lll}1 & 1\end{array}\right)=2.28, p$ 
$=0.024, d=0.45)$. Abstinent patients and current drinkers did not differ in levels of social anxiety, generalized anxiety, or number of comorbid conditions (see Table 2).

\section{Insert Table 2 about here}

\section{Analysis of attentional bias scores}

Kolmogorov Smirnov tests confirmed that attentional bias scores did not differ from normality ( $p$ 's >0.32). Repeated measures analysis of variance (ANOVA) revealed a significant effect of word category on attentional bias $F(4,448)=3.52, p<0.01, n p^{2}=.03$ (see Table 2 for descriptive statistics). One-sample t-tests (against zero $=$ no bias) revealed evidence of significant vigilance towards alcohol-related words $\mathrm{t}(112)=2.25, \mathrm{p}=0.03,95 \% \mathrm{Cl}[1.07$, 16.09], and significant avoidance of depression-related words $\mathrm{t}(112)=2.46, \mathrm{p}<0.01,95 \% \mathrm{Cl}[-$ $15.17,-1.65$ ] across the sample. All other findings were non-significant (t's $(112)<1.02$, p's > $0.30)$.

\section{Effect of current drinking status (abstinence)}

Bias scores were entered into a mixed design ANOVA with drinking status (abstinent vs. drinking) as a between-subject factor and word category as a within-subject factor. Significant main effects of word category $\mathrm{F}(4,444)=3.940, \mathrm{p}=0.004, \eta \mathrm{p}^{2}=.03$ and drinking status $\mathrm{F}(1,111)=4.03, \mathrm{p}<0.05, \eta \mathrm{p}^{2}=.04$ were subsumed under a significant interaction between drinking status and word category, $\mathrm{F}(4,444)=2.53, \mathrm{p}=0.04, \mathrm{np}^{2}=.02$ (Table 2). Patients who were currently drinking displayed a strong attentional bias towards alcohol words $\mathrm{t}(42)=$ 2.695, $\mathrm{p}=0.01,95 \% \mathrm{Cl}[4.46,31.04]$ and social anxiety words $\mathrm{t}(42)=2.84, \mathrm{p}<0.01,95 \%$ $\mathrm{Cl}[3.60,21.36]$ (all other results non-significant (t's $(42)<1.37$, p's $>0.18$ ). Abstinent patients did not display vigilance to alcohol or to anxiety cues (t's $(69)<1.67$ ), but did demonstrate significant avoidance of depression-related stimuli $\mathrm{t}(69)=2.676, \mathrm{p}<0.0195 \% \mathrm{Cl}[-20.38$, 2.97].

\section{Correlations between comorbid mood/anxiety, drinking characteristics and attentional bias}

Across the whole sample the magnitude of attentional bias to alcohol was not correlated with length of drinking history, number of comorbid conditions, severity of anxiety/depression, or number of days abstinent (in the abstinent group). However an increased number of comorbid diagnoses, the level of anxiety, the level of depression, and the duration of alcohol use disorder (years) were all associated with reduced attention towards positive stimuli ( $r$ 's > 0.22 , ps < 0.02). Duration of alcohol use disorder was further associated with increased attention to panic-related stimuli $r=0.26, p<0.01$. In currently abstinent participants there was no correlation between duration of abstinence and attentional bias, mood or anxiety.

\section{Discussion}

We explored attentional biases to alcohol, depression and anxiety related cues, in a 'real world' sample of alcohol dependent patients with high levels of psychiatric co-morbidity. Structured diagnostic interviews found comorbid mental disorders in over $80 \%$ of participants; the mean number of co-morbid conditions being three. Given the level of this comorbidity it was not possible to evaluate the impact on the separate disorders in this study, but it 
emphasizes the need to account for this in experimental studies in clinical populations with alcohol use disorders.

We found a significant attentional bias towards alcohol-related words in a treatment-seeking sample that comprised patients on medication, many of whom were still drinking, and with multiple comorbidities. Sub-group analysis confirmed that attention to alcohol was moderated by drinking status with attentional bias to alcohol only present in those who were still drinking alcohol. Consistent with previous studies we observed marked individual differences in attentional bias (particularly to alcohol-related cues) across the total sample, and within the abstinent and non-abstinent sub-groups, with some patients vigilant for alcohol cues, and others avoidant (Table 2). Beyond drinking status, individual differences in attention to alcohol did not covary with other measures of drinking history, number of previous detoxifications, duration of abstinence, number of comorbid conditions, or comorbid illness severity: suggesting a robust association for current drinking status with alcohol attentional bias.

The amount of error variance (noise) in experimental measures of attentional bias depends on the internal reliability of the task. In our study the VPT measure of attentional bias was most reliable in the non-abstinent group towards alcohol cues (Cronbach's alpha $=0.34$, which is low), suggesting that alcohol did not have a negative effect on VPT performance. This low level of reliability is similar to that observed in other VPT studies of substance-related attentional bias (Ataya et al. 2012a observed alphas ranging from $0.0-0.50$ across 7 VPT studies). Consequently future studies must improve their sensitivity and power by improving the reliability of attentional bias measures, for example through the use of idiosyncratic picture as well as linguistic stimuli, use of non-appetitive control stimuli, and employing eye-movement measures of attentional orienting and disengagement that can profile attentional bias across early to late stages of stimulus processing (see Ataya et al. 2012b and Christiansen et al. $2015 \mathrm{~b}$ for related discussion). Regardless, our findings highlight how laboratory methods and findings in non-dependent populations can translate into clinical field studies that further our understanding of clinically relevant processes and might improve outcomes for patients with alcohol dependence.

Abstinent patients did not show significant attentional bias towards or away from alcohol. This differs from the findings of Vollstadt-Klein et al. who reported avoidance of alcohol cues in a small, highly selected sample of 17 patients with AUD but no additional comorbid axis 1 disorder (Vollstadt-Klein et al., 2009). Despite differences in sample size, the findings of our study and those from Vollstadt-Klein et al. suggest that in early stages of abstinence patients have a tendency to avoid alcohol specific cues, but patients with the additional burden of comorbid axis 1 conditions may be less able to do so.

This study is the first known to investigate depression-specific attentional biases in a sample of patients with alcohol dependence. When compared to healthy populations, depressive symptoms were elevated in abstinent and non-abstinent sub-groups. Abstinent patients had lower levels of depression compared to non-abstinent patients, and significant avoidance of depression-related cues. By contrast patients who were still drinking did not selectively attend 
to or avoid depression-related cues. This suggests there are markedly different cognitiveaffective processes operating in abstinent versus non-abstinent patients with AUD, which may be partly accounted for by group differences in depression severity.

Difficulty in tolerating difficult emotions (such as depressive ruminative thoughts) may contribute to the aetiology and maintenance of alcohol dependence, and our finding that treatment-seeking patients with AUD are avoidant of words with depressive content reflects recent findings of attenuated memory for negatively valenced words in currently depressed alcohol-dependent patients (Fridrici et al., 2014). Similarly the finding that depression severity is associated with reduced attentional bias to positive stimuli is also consistent with the anhedonia often seen in patients with AUD (Hatzigiakoumis et al., 2011). Future experimental research should further clarify interactions between mood, cognition and emotion processing, and examine relationships with clinical outcomes.

There is substantial comorbidity between anxiety disorders (e.g. social anxiety disorder, generalised anxiety disorder, panic disorder) and alcohol dependence (Baldwin et al., 2014). Anxiety disorders develop in late childhood or early adolescence and usually predate the development of an alcohol problem, leading to the suggestion of a 'self-medication' hypothesis (Crum et al., 2012; Crum and Pratt, 2001). Factors related to alcohol dependence, such as alcohol withdrawal, also contribute to patients' self-report of anxiety, and the link between alcohol use and anxiety reduction is reinforced by learning behaviour. By the time patients present to services (as in this sample) having had an alcohol problem for on average 15 years, and 67\% with a comorbid anxiety disorder, it is difficult to separate the various comorbid conditions (Schade et al., 2004). We found strong evidence that increased levels of social and generalized anxiety were associated with reduced attention to positive stimuli, but did not find further associations with attentional bias to anxiety-related stimuli. However duration of alcohol misuse was associated with increased attention to panic cues ( $p<0.01$ ), and in those still drinking there was a strong attentional bias towards social anxiety cues ( $p=$ 0.007): our findings provide some evidence that drinking history and current drinking status are associated with attention towards anxiogenic/threat stimuli in AUD.

This is a large study of patients from a clinical population with the full range of co-morbid conditions anticipated in a group of patients seeking treatment for alcohol dependence. Coexisting psychopathology has been measured and examined rather than excluded. Findings provide evidence of the marked avoidance of depression related cues in patients with AUDs. It also provides strong evidence of an alcohol attentional bias towards alcohol in patients who are still drinking when those with coexisting psychopathology are not excluded. However, the sample is cross-sectional and it remains uncertain whether attentional bias towards alcohol (and social anxiety) cues in current drinkers is caused by their drinking status and will remit with abstinence, or whether it contributes to continued drinking.

Future research should examine whether reduced attention to depressive and anxiogenic material in abstinent patients constitutes an 'adaptive' form of emotion regulation that could be targeted in treatment to sustain abstinence; and whether profiling attentional bias for 
alcohol and emotional information could predict relapse in patients with AUD and comorbid disorders. Longitudinal research is needed to elucidate whether these attentional biases represent static prognostic factors or if they change in response to treatment effects.

\section{Acknowledgements}

Thanks to Bina Nausheen and Katharine Reed for their contributions to the study, to Magda Nowak for secretarial support, to the referring clinical teams, and to participating patients. 


\section{References}

Ataya, A. F., Adams, S., Mullings, E., Cooper, R. M., Attwood, A. S., \& Munafò, M. R. (2012). Internal reliability of measures of substance-related cognitive bias. Drug and Alcohol Dependence, 121(1-2), 148-151. doi:10.1016/j.drugalcdep.2011.08.023

Ataya, A. F., Adams, S., Mullings, E., Cooper, R. M., Attwood, A. S., \& Munafò, M. R. (2012). Methodological considerations in cognitive bias research: The next steps. Drug and Alcohol Dependence, 124(3), 191-192. doi:10.1016/j.drugalcdep.2012.02.008

Baldwin, DS, Anderson, IM, Nutt, DJ, Allgulander, C, Bandelow, B, den Boer, JA, et al. (2014) Evidence-based pharmacological treatment of anxiety disorders, posttraumatic stress disorder and obsessive-compulsive disorder: A revision of the 2005 guidelines from the British Association for Psychopharmacology. J Psychopharmacol 28: 403-439.

Bolton, JM, Robinson, J and Sareen, J (2009) Self-medication of mood disorders with alcohol and drugs in the National Epidemiologic Survey on Alcohol and Related Conditions. J Affect Disord 1 15: 367-375.

Bruce, SE, Yonkers, KA, Otto, MW, Eisen, JL, Weisberg, RB, Pagano, M, et al. (2005) Influence of psychiatric comorbidity on recovery and recurrence in generalized anxiety disorder, social phobia, and panic disorder: a 12-year prospective study. Am J Psychiatry 162: 1179-1187.

Burns, L, Teesson, M and O'Neill, K (2005) The impact of comorbid anxiety and depression on alcohol treatment outcomes. Addiction 100: 787-796.

Christiansen, P, Schoenmakers, TM and Field, M (2015a) Less than meets the eye: Reappraising the clinical relevance of attentional bias in addiction. Addict Behav 44: 43-50.

Christiansen, P., Mansfield, R., Duckworth, J., Field, M., \& Jones, A. (2015b). Internal reliability of the alcohol-related visual probe task is increased by utilising personalised stimuli and eye-tracking. Drug and Alcohol Dependence: 155; 170-174.

Cox, WM, Pothos, EM and Hosier, SG (2007) Cognitive-motivational predictors of excessive drinkers' success in changing. Psychopharmacology 192: 499-510. 
Cranford, J, Nolen-Hoeksema, S and Zucker, R (2011) Alcohol involvement as a function of co-occurring alcohol use disorders and major depressive episode: Evidence from the National Epidemiologic Survey on Alcohol and Related Conditions. Drug Alcohol Depend 117: 145-151.

Crum, RM, La Flair, L, Storr, CL, Green, KM, Stuart, EA, Alvanzo, AAH, et al. (2013) Reports of drinking to self-medicate anxiety symptoms: Iongitudinal assessment for subgroups of individuals with alcohol dependence Depress Anxiety 30: 174-83.

Crum, RM and Pratt, LA (2001) Risk of heavy drinking and alcohol use disorders in social phobia: a prospective analysis. Am J Psychiatry 158: 1693-1700.

Donaldson, C, Lam, D and Mathews, A (2007) Rumination and attention in major depression. Behav Res Ther 45: 2664-2678.

Fadardi, JS and Cox, WM (2009) Reversing the sequence: reducing alcohol consumption by overcoming alcohol attentional bias. Drug Alcohol Depend. 101: 137-145.

Field, M, Duka, T, Eastwood, B, Child, R, Santarcangelo, M and Gayton, M (2007) Experimental manipulation of attentional biases in heavy drinkers: do the effects generalise? Psychopharmacology (Berl) 192: 593-608.

Field, M and Eastwood, B (2005) Experimental manipulation of attentional bias increases the motivation to drink alcohol. Psychopharmacology (Berl) 183: 350-357.

Field, M, Mogg, K, Mann, B, Bennett, GA and Bradley, BP (2013) Attentional biases in abstinent alcoholics and their association with craving. Psychol Addict Behav 27: $71-80$.

Fridrici, C, Driessen, M, Wingenfeld, K, Kremer, G, Kißler, J and Beblo, T (2014) Investigating biases of attention and memory for alcohol-related and negative words in alcohol-dependents with and without major depression after dayclinic treatment. Psychiatry Res 218: 311-218.

Grant, B, Stinson, FS, Dawson, DA and et al. (2004) Prevalence and co-occurrence of substance use disorders and independentmood and anxiety disorders: 
Results from the national epidemiologic survey on alcohol and relatedconditions. Arch Gen Psychiatry 61: 807-816.

Grant, DM and Beck, JG (2006) Attentional biases in social anxiety and dysphoria: does comorbidity make a difference? J Anxiety Disord 20: 520-529.

Hatzigiakoumis, DS, Martinotti, G, Di Giannantonio, M and Janiri, L (2011) Anhedonia and substance dependence: clinical correlates and treatment options. Front Psychiatry 2: 10.

Kaplan, JS, Erickson, K, Luckenbaugh, DA, Weiland-Fiedler, P, Geraci, M, Sahakian, BJ, et al. (2006) Differential performance on tasks of affective processing and decision-making in patients with Panic Disorder and Panic Disorder with comorbid Major Depressive Disorder. J Affect Disord 95: 165-171.

Kessler, RC, Chiu, WT, Demler, O, Merikangas, KR and Walters, EE (2005) Prevalence, severity, and comorbidity of 12-month DSM-IV disorders in the National Comorbidity Survey Replication. Arch Gen Psychiatry 62: 61 7-627.

Koster, E, Crombez, G, Verschuere, B and De Houwer, J (2004) Selective attention to threat in the dot probe paradigm: differentiating vigilance and difficulty to disengage. Behav Res Ther 42: 1183-1192.

Lecrubier, Y, Sheehan, DV, Weiller, E, Amorim, P, Bonora, I, Sheehan, KH, et al. (1997) The Mini International Neuropsychiatric Interview (MINI). A short diagnostic structured interview: reliability and validity according to the CIDI. Eur Psychiatry 12: 224-231.

Liebowitz (1987) Social Phobia. Mod Probl Pharmacopsychiatry 22: 141-173.

Lingford-Hughes, A, Welch, S, Peters, L and Nutt, D (2012) Evidence-based guidelines for the pharmacological management of substance abuse, harmful use, addiction and comorbidity: recommendations from BAP. Journal of Psychopharmacology. 26(7):899-952.

Loeber, S, Vollstadt-Klein, S, von der, GC, Flor, H, Mann, K and Kiefer, F (2009) Attentional bias in alcohol-dependent patients: the role of chronicity and executive functioning. Addict Biol 14: 194-203. 
MacLeod, C, Mathews, A and Tata, P (1986) Attentional bias in emotional disorders. J Abnorm Psychology 95: 15-20.

Moore DJ, KE, Eccleston Ch. (2009) Identifying experimental methods to determine the effect of pain on attention: a review of pain, caffeine, alcohol and nicotine studies. Hum Psychopharmacol 24: 601-618.

Musa, C, Lepine, JP, Clark, DM, Mansell, W and Ehlers, A (2003) Selective attention in social phobia and the moderating effect of a concurrent depressive disorder. Behav Res Ther 41: 1043-1054.

Noel, X, Colmant, M, Van Der, LM, Bechara, A, Bullens, Q, Hanak, C, et al. (2006) Time course of attention for alcohol cues in abstinent alcoholic patients: the role of initial orienting. Alcohol Clin Exp Res 30: 1871-1877.

Robins, L, Wing, J, Wittchen, H, Helzer, J, Babor, T, Burke, J, et al. (1988) The Composite International Diagnostic Interview. An epidemiologic Instrument suitable for use in conjunction with different diagnostic systems and in different cultures. Arch Gen Psychiatry 45: 1069-1077.

Schade, A, Marquenie, LA, Van Balkom, AJLM, Koeter, M, Beurs, E, Brink, WVD, et al. (2004) Alcohol-dependent patients withcomorbid phobic disorders: a comparison between comorbid patients, pure alcohol-dependent and pure phobic patients. Alcohol Alcohol 39: 241-246.

Sheehan, D, Lecrubier, Y, Sheehan, K, Amorim, P, Janavs, J, Weiller, E, et al. (1998) The Mini-International Neuropsychiatric Interview (M.I.N.I.): the development and validation of a structured diagnostic psychiatric interview for DSM-IV and ICD-10. J Clin Psychiatry 59 Suppl 20: 22-33.

Sinclair, J, Nausheen, B, Garner, M and Baldwin, D (2010) Attentional biases in clinical populations with alcohol use disorders: is co-morbidity ignored? . Hum Psychopharmacol 25: 515-524.

Stormark, KM, Field, NP, Hugdahl, K and Horowitz, M (1997) Selective processing of visual alcohol cues in abstinent alcoholics: an approach-avoidance conflict? Addict Behav 22: 509-519. 
Townshend, JM and Duka, T (2007) Avoidance of alcohol-related stimuli in alcoholdependent inpatients. Alcohol Clin Exp Res 31: 1349-1357.

Vollstadt-Klein, S, Loeber, S, von der, GC, Mann, K and Kiefer, F (2009) Avoidance of alcohol-related stimuli increases during the early stage of abstinence in alcohol-dependent patients. Alcohol Alcohol 44: 458-463.

Zigmond AS, SR (1983) The hospital anxiety and depression scale. Acta Psychiatr Scand 67: 361-370. 
Table 1

Sample Characteristics

\begin{tabular}{|c|c|c|}
\hline & $\mathbf{n}$ & $\begin{array}{l}\text { \% of Sample } \\
\text { (SD/ IQR)* }\end{array}$ \\
\hline Mean age in years (standard deviation) & 44.4 & $(11.2)$ \\
\hline \multicolumn{3}{|l|}{ Demographics $N=113$} \\
\hline \multicolumn{3}{|l|}{ Gender } \\
\hline Female & 42 & $37.2 \%$ \\
\hline Male & 71 & $62.8 \%$ \\
\hline \multicolumn{3}{|l|}{ Marital Status } \\
\hline Married/ Living as married & 31 & $27.4 \%$ \\
\hline Single & 43 & $38.1 \%$ \\
\hline Divorced/ separated/ widowed & 39 & $34.5 \%$ \\
\hline \multicolumn{3}{|l|}{ Education } \\
\hline Below GCSE & 20 & $17.7 \%$ \\
\hline GCSE/ O Level & 48 & $42.5 \%$ \\
\hline Above GCSE (A level, degree) & 45 & $39.8 \%$ \\
\hline \multicolumn{3}{|l|}{ Employment } \\
\hline Employed & 25 & $22.1 \%$ \\
\hline Benefits (Unemployed/ Disability Allowance) & 72 & $63.7 \%$ \\
\hline Other & 16 & $14.2 \%$ \\
\hline \multicolumn{3}{|l|}{ Alcohol History $\mathrm{N}=113$} \\
\hline Family history of AUD & 76 & $67.3 \%$ \\
\hline Median years of AUD (IQR) & 15.0 & $(6-24)$ \\
\hline Median number of previous detoxifications (IQR) & 1.00 & $(0-2)$ \\
\hline \multicolumn{3}{|l|}{ Current drinking status } \\
\hline Abstinent & 70 & $61.9 \%$ \\
\hline Still drinking & 43 & $38.1 \%$ \\
\hline \multicolumn{3}{|l|}{ Current drinkers $(\mathrm{N}=43)$ : } \\
\hline Median units per day (IQR) & 15.0 & $(9-23)$ \\
\hline Median number of days of alcohol use per week (IQR) & 7.0 & $(3-7)$ \\
\hline Median units per week (IQR) & 70 & $(24-140)$ \\
\hline \multicolumn{3}{|l|}{ Diagnoses on MINI } \\
\hline Alcohol use disorder -AUD ( $\mathrm{N}=113)$ & 111 & $98.2 \%$ \\
\hline One or more comorbid diagnoses $(\mathrm{N}=107)$ & 92 & $81.4 \%$ \\
\hline Depression spectrum (Major depressive disorder \& dysthymia) $(\mathrm{N}=111)$ & 76 & $67.9 \%$ \\
\hline Hypomania spectrum (Mania \& hypomania) $(\mathrm{N}=111)$ & 43 & $38.1 \%$ \\
\hline Other Substance use disorder (excluding AUD) $(\mathrm{N}=109)$ & 19 & $16.8 \%$ \\
\hline $\begin{array}{l}\text { Anxiety (panic disorder, agoraphobia, social phobia, OCD, PTSD \& GAD) } \\
(\mathrm{N}=113)\end{array}$ & 76 & $67.3 \%$ \\
\hline
\end{tabular}

*SD/ IQR. Standard deviation of mean/or Interquartile range of median 
Table 2:

Effect of Current drinking status on attentional bias and co-existing psychopathology

\begin{tabular}{|c|c|c|c|c|c|c|}
\hline & \multicolumn{2}{|c|}{ Overall $(\mathrm{N}=113)$} & \multicolumn{2}{|c|}{ Abstinent $(n=70)$} & \multicolumn{2}{|c|}{ Currently drinking $(n=43)$} \\
\hline & $\mathrm{M}$ & SD & $\mathrm{M}$ & SD & $\mathrm{M}$ & SD \\
\hline Depression (HADS) & $6.8^{*}$ & 4.9 & $5.9^{*, a}$ & 4.7 & $8.3^{*, b}$ & 4.9 \\
\hline Anxiety (HADS) & $10.2^{*}$ & 5.1 & $10.0 *$ & 4.9 & $10.5^{*}$ & 5.3 \\
\hline Social anxiety (LSAS) & $54.1 *$ & 35.9 & $57.2 *$ & 37.3 & $48.8^{*}$ & 30.4 \\
\hline $\begin{array}{l}\text { No. comorbid } \\
\text { conditions }\end{array}$ & 3.0 & 2.3 & 3.0 & 2.5 & 2.7 & 2.0 \\
\hline Comorbid conditions & $\mathrm{N}$ & $\%$ & $\mathrm{n}$ & $\%$ & $\mathrm{n}$ & $\%$ \\
\hline $\begin{array}{c}\text { Major depression } \\
\text { /dysthymia }\end{array}$ & 76 & 68 & 45 & 65 & 31 & 74 \\
\hline Mania/hypomania & 43 & 38 & 31 & 44 & 12 & 28 \\
\hline Anxiety disorder & 76 & 67 & 49 & 70 & 27 & 62 \\
\hline $\begin{array}{c}\text { Other Substance } \\
\text { Disorder }\end{array}$ & 19 & 17 & 10 & 14 & 9 & 21 \\
\hline Alcohol use? & $M$ & SD & $M$ & SD & $M$ & SD \\
\hline $\begin{array}{c}\text { Duration of misuse } \\
\text { (years) }\end{array}$ & 15.4 & 10.2 & $17.1^{\mathrm{a}}$ & 10.4 & $12.7^{b}$ & 9.2 \\
\hline Days Abstinent & - & - & 90.5 & 117.1 & - & - \\
\hline Attention Bias (ms) & $\mathrm{M}$ & SD & $M$ & SD & $M$ & SD \\
\hline Alcohol & $8.5^{*}$ & $(40.3)$ & $2.9^{a}$ & (37.6) & $17.8^{*, \mathrm{~b}}$ & $(43.2)$ \\
\hline Depression & $-8.4 *$ & (36.3) & $-11.7^{*}$ & (36.5) & -3.1 & (35.6) \\
\hline Panic & 1.7 & $(34.8)$ & 2.7 & (33.4) & 0.1 & $(37.3)$ \\
\hline Positive & -3.6 & (37.4) & -1.4 & (39.4) & -7.2 & (34.1) \\
\hline Social Anxiety & 0.2 & (35.1) & $-7.3^{a}$ & (36.7) & $12.5^{*, b}$ & (28.9) \\
\hline
\end{tabular}

Note * = questionnaire score significantly differs from healthy population norm (Crawford et al. 2001); or attentional bias score significantly differs from zero (no bias). Values with different superscripts $(\mathrm{a}, \mathrm{b})$ significantly differ between abstinent and non-abstinent patient groups. 